JOURNAL OF THE

AMERICAN MATHEMATICAL SOCIETY

Volume 17, Number 1, Pages 1-18

S 0894-0347(03)00444-2

Article electronically published on September 26, 2003

\title{
WIENER'S LEMMA FOR TWISTED CONVOLUTION AND GABOR FRAMES
}

\author{
KARLHEINZ GRÖCHENIG AND MICHAEL LEINERT
}

\section{INTRODUCTION}

Wiener's Lemma states that if a periodic function $f$ has an absolutely convergent Fourier series that never vanishes, then $1 / f$ also has an absolutely convergent Fourier series. An equivalent formulation that is more suitable for generalization considers the convolution operator $C_{\mathbf{a}} \mathbf{c}=\mathbf{a} * \mathbf{c}$ acting on $\ell^{p}\left(\mathbb{Z}^{d}\right)$. In this case, Wiener's Lemma states the following:

If $\mathbf{a} \in \ell^{1}\left(\mathbb{Z}^{d}\right)$ and if $C_{\mathbf{a}}$ is invertible as an operator on $\ell^{2}\left(\mathbb{Z}^{d}\right)$, then a is invertible in $\ell^{1}\left(\mathbb{Z}^{d}\right)$ and hence the inverse operator is of the form $C_{\mathbf{a}}^{-1}=C_{\mathbf{b}}$ for some $\mathbf{b} \in \ell^{1}\left(\mathbb{Z}^{d}\right)$.

As a consequence, $C_{\mathbf{a}}$ is invertible and bounded on all $\ell^{p}\left(\mathbb{Z}^{d}\right)$ for $1 \leq p \leq \infty$ simultaneously.

In this article we study several non-commutative generalizations of Wiener's Lemma and their application to Gabor theory. The paper is divided into two parts: the first part (Sections 2 and 3 ) is devoted to abstract harmonic analysis and extends Wiener's Lemma to twisted convolution. The second part (Section 4) is devoted to the theory of Gabor frames, specifically to the design of dual windows with good time-frequency localization. In particular, we solve a conjecture of Janssen, Feichtinger and one of us [17, 18, 9].

These two topics appear to be completely disjoint, but they are not. The solution of the conjectures about Gabor frames is an unexpected application of methods from non-commutative harmonic analysis to application-oriented mathematics. It turns out that the connection between twisted convolution and the Heisenberg group and the theory of symmetric group algebras are precisely the tools needed to treat the problem motivated by signal analysis.

To be more concrete, we formulate some of our main results first and will deal with the details and the technical background later.

Received by the editors July 1, 2001.

2000 Mathematics Subject Classification. Primary 22D25, 42C15; Secondary 22E25, 47B38, $47 \mathrm{C} 15$.

Key words and phrases. Twisted convolution, Heisenberg group, Wiener's Lemma, symmetric group algebra, Gabor frame, modulation space, window design, invertibility of operators.

The first author acknowledges partial support by the Austrian Science Foundation (FWF) under project no. P14485-MAT. 
Twisted Convolution. Given $\theta>0$, we study the twisted convolution of two sequences $\mathbf{a}=\left(a_{k l}\right)_{k, l \in \mathbb{Z}^{d}}$ and $\mathbf{b}=\left(b_{k l}\right)_{k, l \in \mathbb{Z}^{d}}$ on $\mathbb{Z}^{2 d}$, which is defined to be

$$
\left(\mathbf{a} \natural_{\theta} \mathbf{b}\right)(m, n)=\sum_{k, l \in \mathbb{Z}^{d}} a_{k l} b_{m-k, n-l} e^{2 \pi i \theta(m-k) \cdot l} .
$$

Since

$$
\left|\mathbf{a} \hbar_{\theta} \mathbf{b}(m, n)\right| \leq(|\mathbf{a}| *|\mathbf{b}|)(m, n)
$$

the convolution relations of Young's Theorem carry over to twisted convolution. In particular, we have $\ell^{1} \natural_{\theta} \ell^{p} \subseteq \ell^{p}$ for $1 \leq p \leq \infty$.

We will prove several versions of the following statement, which is Wiener's Lemma for twisted convolution.

Theorem 1.1. Assume that $\mathbf{a} \in \ell^{1}\left(\mathbb{Z}^{2 d}\right)$ and that the (twisted) convolution operator $L_{\mathbf{a}}$, defined by $L_{\mathbf{a}} \mathbf{c}=\mathbf{a} \hbar_{\theta} \mathbf{c}$, is invertible on $\ell^{2}\left(\mathbb{Z}^{2 d}\right)$. Then $\mathbf{a}$ is invertible in $\ell^{1}\left(\mathbb{Z}^{2 d}\right)$ and so $L_{\mathbf{a}}^{-1}=L_{\mathbf{b}}$ for some $\mathbf{b} \in \ell^{1}\left(\mathbb{Z}^{2 d}\right)$. Consequently $L_{\mathbf{a}}$ is invertible simultaneously on all $\ell^{p}\left(\mathbb{Z}^{2 d}\right)$ for $1 \leq p \leq \infty$.

If $\theta \in \mathbb{Z}$, then $\natural_{\theta}$ coincides with ordinary convolution, and Theorem 1.1 is just the classical Wiener Lemma.

If $\theta \in \mathbb{Q}$, then commutative methods by means of a suitable decomposition of the convolution operator $L_{\mathbf{a}}$ (Lemma 2.4) can be applied to derive Theorem 1.1 An equivalent form of Theorem 1.1 for the "rational case" $\theta \in \mathbb{Q}$ is proved in [14. Ch. 13].

In the "irrational case" $\theta \notin \mathbb{Q}$ commutative methods break down completely and Theorem 1.1 was unproven so far. We prove Theorem 1.1 for all values of $\theta$ and also for weighted $\ell^{1}$-algebras with methods from abstract harmonic analysis. The key is to relate twisted convolution to the ordinary convolution on an associated Heisenberg group and to make use of the fact that the group algebra $L^{1}(G)$ of a nilpotent group is a symmetric Banach $*$-algebra.

Time-Frequency Analysis, Short-Time Fourier Transform, and Gabor Frames. The goal of time-frequency analysis is to understand the properties of functions or distributions simultaneously in time and frequency (physicists use the term phase space analysis for this goal). We refer to [11] and [14 for a mathematical introduction to time-frequency analysis and to [10] for more advanced aspects.

The main tool is the short-time Fourier transform (STFT), also known under the names radar ambiguity function, coherent state transform, or cross Wigner distribution. Let $T_{x} f(t)=f(t-x)$ denote the translation operator on $\mathbb{R}^{d}$ and $M_{\omega} f(t)=e^{2 \pi i \omega \cdot t} f(t)$ be the modulation operator for $x, \omega, t \in \mathbb{R}^{d}$. Then the STFT of a function $f$ on $\mathbb{R}^{d}$ with respect to a fixed window $g$ is defined as

$$
V_{g} f(x, \omega)=\int_{\mathbb{R}^{d}} f(t) \bar{g}(t-x) e^{-2 \pi i \omega \cdot t} d t=\left\langle f, M_{\omega} T_{x} g\right\rangle .
$$

Note that

$$
V_{g} f(x, \omega)=\left\langle\hat{f}, T_{\omega} M_{x} \hat{g}\right\rangle=e^{-2 \pi i x \cdot \omega} V_{\hat{g}} \hat{f}(\omega,-x) .
$$

If both $g$ and $\hat{g}$ are well localized, e.g., if $g \in \mathcal{S}\left(\mathbb{R}^{d}\right)$, then $V_{g} f(x, \omega)$ measures the magnitude of $f$ in a neighborhood of $x$ and of $\hat{f}$ in a neighborhod of $\omega$. For practical 
and numerical purposes one prefers a discrete version of the STFT and aims for series expansions of the form

$$
f=\sum_{k, l \in \mathbb{Z}^{d}}\left\langle f, T_{\alpha k} M_{\beta l} g\right\rangle T_{\alpha k} M_{\beta l} \gamma .
$$

Once again, the coefficient $c_{k l}=\left\langle f, T_{\alpha k} M_{\beta l} g\right\rangle$ describes the combined timefrequency behavior of $f$ at a point $(\alpha k, \beta l)$ in the "time-frequency plane" $\mathbb{R}^{2 d}$. A pair $(g, \gamma)$ as in (1.5) is called a pair of dual windows.

To construct the so-called Gabor expansions as in (1.5), one starts with a single window $g \in L^{2}\left(\mathbb{R}^{d}\right)$ and fixed lattice parameters $\alpha, \beta>0$ and studies the spectrum of the associated Gabor frame operator $S=S_{g, \alpha, \beta}$. This operator imitates an orthogonal expansion and is defined as

$$
S f=\sum_{k, l \in \mathbb{Z}^{d}}\left\langle f, T_{\alpha k} M_{\beta l} g\right\rangle T_{\alpha k} M_{\beta l} g .
$$

Since $\langle S f, f\rangle=\sum_{k, l \in \mathbb{Z}^{d}}\left|\left\langle f, T_{\alpha k} M_{\beta l} g\right\rangle\right|^{2} \geq 0, S$ is a positive operator. If $S$ has a bounded inverse on $L^{2}\left(\mathbb{R}^{d}\right)$, then the function $\gamma=S^{-1} g$ is well defined in $L^{2}\left(\mathbb{R}^{d}\right)$ and is a dual window in the sense of (1.5)). To see this, we observe that $S$ and $S^{-1}$ commute with all time-frequency shifts $T_{\alpha k} M_{\beta l}$; therefore, we obtain the factorization

$$
\begin{aligned}
f & =S^{-1} S f=\sum_{k, l \in \mathbb{Z}^{d}}\left\langle f, T_{\alpha k} M_{\beta l} g\right\rangle T_{\alpha k} M_{\beta l} S^{-1} g \\
& =S S^{-1} f=\sum_{k, l \in \mathbb{Z}^{d}}\left\langle f, T_{\alpha k} M_{\beta l} S^{-1} g\right\rangle T_{\alpha k} M_{\beta l} g .
\end{aligned}
$$

Thus we can take $\gamma=S^{-1} g$ to establish the Gabor expansion (1.5). Furthermore

$$
\|f\|_{2} \asymp\left(\sum_{k, l \in \mathbb{Z}^{d}}\left|\left\langle f, T_{\alpha k} M_{\beta l} g\right\rangle\right|^{2}\right)^{1 / 2} \asymp\left(\sum_{k, l \in \mathbb{Z}^{d}}\left|\left\langle f, T_{\alpha k} M_{\beta l} \gamma\right\rangle\right|^{2}\right)^{1 / 2}
$$

for all $f \in L^{2}\left(\mathbb{R}^{d}\right)$, where $\asymp$ denotes the equivalence of norms. Condition (1.9) is usually expressed by saying that each of the sets $\left\{T_{\alpha k} M_{\beta l} g: k, l \in \mathbb{Z}^{d}\right\}$ and $\left\{T_{\alpha k} M_{\beta l} \gamma: k, l \in \mathbb{Z}^{d}\right\}$ is a frame for $L^{2}\left(\mathbb{R}^{d}\right)$.

The inversion of the Gabor frame operator $S_{g, \alpha, \beta}$ on $L^{2}\left(\mathbb{R}^{d}\right)$ is well understood; see [3], 31]. Vaguely formulated, these results state that $S_{g, \alpha, \beta}$ is invertible for any window $g$ satisfying a mild decay condition and for $\alpha, \beta>0$ small enough. On the other hand, for such windows the density condition $\alpha \beta<1$ is necessary for the invertibility of $S$ on $L^{2}\left(\mathbb{R}^{d}\right)$ (see [3] or [14, Cor. 8.4.3]).

For a genuine time-frequency analysis, however, the pure $L^{2}$-theory is insufficient. For instance, $\left\{T_{k} M_{l} \chi_{[0,1]^{d}}: k, l \in \mathbb{Z}^{d}\right\}$ is even an orthonormal basis of $L^{2}\left(\mathbb{R}^{d}\right)$, but since $\widehat{\chi_{[0,1] d}}$ decays slowly, the coefficients $\left\langle f, T_{k} M_{l} \chi\right\rangle$ do not furnish any frequency localization, and thus it is not possible to distinguish a smooth function from a "rough" function by looking only at the coefficients in (1.5).

For a better description of the properties of a function in both time and frequency, we need a pair of dual windows $(g, \gamma)$ that satisfy (1.5) and possess good decay properties and smoothness. In terms of the construction $\gamma=S^{-1} g$, these properties of $(g, \gamma)$ are equivalent to the invertibility of $S$ on other function spaces.

A major result in this direction was proved by Janssen [17, Prop. 5.5]: If $S_{g, \alpha, \beta}$ is invertible on $L^{2}\left(\mathbb{R}^{d}\right)$ and if $g \in \mathcal{S}\left(\mathbb{R}^{d}\right)$, then $\gamma=S^{-1} g$ is in $\mathcal{S}\left(\mathbb{R}^{d}\right)$ as well. 
In order to obtain more detailed information about window construction we use the decay of the short-time Fourier transform as a measure for time-frequency concentration.

Fix a symmetric weight function $v \geq 1$ on $\mathbb{R}^{2 d}$, and let $\phi(x)=e^{-\pi x^{2}}$ be the Gaussian on $\mathbb{R}^{d}$. We say that $f$ is in the modulation space $M_{v}^{1}$, if

$$
\|f\|_{M_{v}^{1}}=\int_{\mathbb{R}^{2 d}}\left|V_{\phi} f(z)\right| v(z) d z<\infty .
$$

This condition implies decay of the short-time Fourier transform and can be translated into more explicit smoothness and decay properties of $f[13$. If $v \equiv 1$, then $M_{v}^{1}$ coincides with Feichtinger's algebra $S_{0}$, which plays already an important role in commutative harmonic analysis and in Gabor analysis [7, 10].

Our main result on window design is the following.

Theorem 1.2. Assume that $v$ is a positive, continuous and radial function on $\mathbb{R}^{2 d}$ that satisfies $v(0)=1, v\left(r_{1}+r_{2}\right) \leq v\left(r_{1}\right) v\left(r_{2}\right), r_{1}, r_{2} \in \mathbb{R}^{2 d}$, and such that $\lim _{n \rightarrow \infty} v(n r)^{1 / n}=1$ for all $r \in \mathbb{R}^{2 d}$. If $g \in M_{v}^{1}$ and if $S_{g, \alpha, \beta}$ is invertible on $L^{2}\left(\mathbb{R}^{d}\right)$, then $S_{g, \alpha, \beta}$ is invertible on $M_{v}^{1}$ and thus $\gamma=S^{-1} g \in M_{v}^{1}$.

[The conditions on the weight $v$ can be relaxed to so-called sub-exponential weights; see Definition 2.12.]

This statement has a structure similar to Theorem 1.1. The invertibility on a Hilbert space and the "smoothness" of the "symbol" already imply the invertibility on other function spaces.

For weights of polynomial growth and $\alpha \beta \in \mathbb{Q}$, Theorem 1.2 was proved in 9 . Thm. 3.4]; see also [14, Ch. 13] for variations. The rationality condition $\alpha \beta \in \mathbb{Q}$ occurs frequently in time-frequency analysis, because in this case the Gabor frame operator possesses an additional structure and can be investigated with commutative methods [2, [9], 18, [29, 32.

A priori there is no reason why Theorem 1.2 should hold only for rational values of $\alpha \beta$. It was conjectured in [9] Rem. 3.2] and in [17], [18] that Theorem 1.2 holds also in the so-called "irrational case", but so far this problem had remained open. This paper proves the conjecture.

1.1. The Rotation Algebra. To give a glimpse of what is involved, let $C^{*}(\alpha, \beta)$ be the $C^{*}$-algebra generated by the unitary operators $\left\{T_{\alpha k} M_{\beta l}: k, l \in \mathbb{Z}^{d}\right\}$ for fixed $\alpha, \beta>0$. This is a representation of the so-called rotation algebra [5]. If $\alpha \beta \in \mathbb{Q}$, then its structure is so easy to understand that it is given as an exercise in 5 . If $\alpha \beta \notin \mathbb{Q}$, then $C^{*}(\alpha, \beta)$ is a simple algebra, but its detailed structure is very complex as is manifested by the deep investigations in [6], 26], 27]. One may expect that a proof of Theorem 1.2 in the irrational case must rely on deeper resources than Fourier transform methods.

Since the Gabor frame operator $S$ commutes with all time-frequency shifts $T_{\alpha k} M_{\beta l}$, it is in the commutant of $C^{*}(\alpha, \beta)$. It is well known that the commutant is generated by the time-frequency shifts $T_{k / \beta} M_{l / \alpha}$; therefore, we may expect $S$ to be a limit of finite linear combinations of the $T_{k / \beta} M_{l / \alpha}$. Precisely, Janssen 17] and Daubechies, Landau, and Landau [4] showed that

$$
S_{g, \alpha, \beta} f=(\alpha \beta)^{-d} \sum_{k, l \in \mathbb{Z}^{d}}\left\langle g, T_{\frac{k}{\beta}} M_{\frac{l}{\alpha}} g\right\rangle T_{\frac{k}{\beta}} M_{\frac{l}{\alpha}} f .
$$


For the better understanding of the deeper properties of $S$, it is natural to study the (Banach) subalgebra of $C^{*}(\alpha, \beta)$ consisting of absolutely convergent series of time-frequency shifts.

Definition 1.3. Given $\alpha, \beta>0$ and a submultiplicative weight $v$ (see (2.15), the operator algebra $\mathcal{A}_{v}(\alpha, \beta)$ is defined to be

$$
\mathcal{A}_{v}(\alpha, \beta)=\left\{A \in \mathcal{B}\left(L^{2}\left(\mathbb{R}^{d}\right)\right): A=\sum_{k, l \in \mathbb{Z}^{d}} a_{k l} T_{\alpha k} M_{\beta l}, \mathbf{a} \in \ell_{v}^{1}\left(\mathbb{Z}^{2 d}\right)\right\}
$$

with (semi-) norm $\|A\|_{\mathcal{A}_{v}}=\|\mathbf{a}\|_{1, v}$.

Let $\pi$ be the mapping from $\ell_{v}^{1}\left(\mathbb{Z}^{2 d}\right)$ into $\mathcal{A}_{v}(\alpha, \beta)$ defined by

$$
\pi(\mathbf{a})=\sum_{k, l \in \mathbb{Z}^{d}} a_{k l} T_{\alpha k} M_{\beta l} .
$$

Then $\mathcal{A}_{v}(\alpha, \beta)=\pi\left(\ell_{v}^{1}\left(\mathbb{Z}^{2 d}\right)\right)$. We will show in Lemma 3.3 that $\pi$ is one-to-one. Consequently $\|A\|_{\mathcal{A}_{v}}$ is a norm on $\mathcal{A}_{v}(\alpha, \beta)$, and $\mathcal{A}_{v}(\alpha, \beta)$ is a dense Banach subalgebra of the $C^{*}$-algebra $C^{*}(\alpha, \beta)$.

Following Janssen[17], we make a key observation: set $\theta=\alpha \beta$; then

$$
\pi(\mathbf{a}) \pi(\mathbf{b})=\pi\left(\mathbf{a} \natural_{\theta} \mathbf{b}\right)
$$

and thus $\pi$ is an algebra homomorphism from $\ell_{v}^{1}\left(\mathbb{Z}^{2 d}\right)$ onto $\mathcal{A}_{v}(\alpha, \beta)$.

Once the relation between the Gabor frame operator and twisted convolution is understood, it is plausible that we may apply the abstract result, namely Wiener's Lemma for twisted convolution, to the concrete problem of inverting the Gabor frame operator. Using this strategy, we will solve the "irrational case" of Gabor frames and solve the conjectures in [17, [9].

In the context of operator algebras the following variation of Wiener's Lemma, which is equivalent to Theorem 1.1, might be of interest.

Theorem 1.4. If $A \in \mathcal{A}_{v}(\alpha, \beta)$ is invertible in $C^{*}(\alpha, \beta)$, then $A^{-1} \in \mathcal{A}_{v}(\alpha, \beta)$.

Turning Wiener's Lemma into a definition, Naimark [22, p. 203] calls a nested pair $\mathcal{A} \subseteq \mathcal{B}$ of Banach algebras with a common identity a Wiener pair, if every element of $\mathcal{A}$ that is invertible in the larger algebra $\mathcal{B}$ is also invertible in $\mathcal{A}$. So Theorem 1.4 states that the (non-commutative) algebras $\mathcal{A}_{v}(\alpha, \beta)$ and $C^{*}(\alpha, \beta)$ form a Wiener pair.

The paper is organized as follows: In Section 2 we first provide the necessary background on twisted convolution and its connection to the Heisenberg group; then we discuss the concept of symmetric group algebras. The main result (Theorem 1.1) follows by a combination of these ideas.

In Section 3 we investigate the Banach algebra of operators $\mathcal{A}_{v}(\alpha, \beta)$ and prove Wiener's Lemma for $\mathcal{A}_{v}(\alpha, \beta)$ (Theorem 1.4).

In Section 4 we apply these abstract results to Gabor frames and prove the existence of good windows in terms of time-frequency concentration. For the applications of Gabor theory in signal analysis these are probably the most interesting results of this paper. 


\section{Analysis of twisted CONVOlution}

To make $\ell^{1}\left(\mathbb{Z}^{2 d}\right)$ into an involutive Banach algebra, we introduce an involution operation that is compatible with $\natural_{\theta}$ in the following way:

$$
a_{k l}^{*}=\overline{a_{-k,-l}} e^{2 \pi i \theta k \cdot l} .
$$

Lemma 2.1. $\ell^{1}\left(\mathbb{Z}^{2 d}\right)$ is an involutive Banach algebra under the twisted convolution $\natural_{\theta}$ and the involution *.

Obviously, if $\theta^{\prime}-\theta \in \mathbb{Z}$, then the twisted convolutions $\natural_{\theta}$ and $\natural_{\theta^{\prime}}$ are identical. Clearly, $\natural_{\theta}$ is commutative if and only if $\theta \in \mathbb{Z}$. In dimension $d=1$ it is known that (a) for $\theta \in(0,1 / 2]$ none of the algebras $\left(\ell^{1}\left(\mathbb{Z}^{2 d}\right), \natural_{\theta}\right)$ are isomorphic, and that (b) for irrational $\theta \notin \mathbb{Q}$ the algebra $\left(\ell^{1}\left(\mathbb{Z}^{2 d}\right)\right.$, $\left.\natural_{\theta}\right)$ is simple [5].

2.1. Heisenberg-Type Groups. Twisted convolution is closely related to ordinary convolution in the group algebra of an associated Heisenberg group.

Definition 2.2. Given $\theta>0$, we define a multiplication $\cdot \theta$ on $\mathbb{Z}^{d} \times \mathbb{Z}^{d} \times \mathbb{T}$. Let $k, l, m, n \in \mathbb{Z}^{d}$ and $\sigma, \tau \in \mathbb{T}=\{z \in \mathbb{C}:|z|=1\}$; then

$$
(k, l, \sigma) \cdot \theta(m, n, \tau)=\left(k+m, l+n, \sigma \tau e^{2 \pi i \theta l \cdot m}\right) .
$$

The multiplication $\cdot_{\theta}$ defines a group structure on $\mathbb{Z}^{d} \times \mathbb{Z}^{d} \times \mathbb{T}$. We shall denote the resulting group by $\mathbb{H}_{\theta}$. Since $\{0\} \times \mathbb{T}$ is contained in the center of $\mathbb{H}_{\theta}$ and $\mathbb{H}_{\theta} /(\{0\} \times \mathbb{T}) \cong \mathbb{Z}^{2 d}$ is abelian, $\mathbb{H}_{\theta}$ is nilpotent and resembles a quotient of the standard Heisenberg group.

The Haar measure $d \lambda$ on $\mathbb{H}_{\theta}=\mathbb{Z}^{d} \times \mathbb{Z}^{d} \times \mathbb{T}$ is given explicitly by $\int F d \lambda=$ $\sum_{k \in \mathbb{Z}^{d}} \sum_{l \in \mathbb{Z}^{d}} \int_{\mathbb{T}} F(k, l, \tau) d \tau$. The group convolution $*_{\theta}$ and $L^{p}\left(\mathbb{H}_{\theta}\right)$ are defined with respect to this measure; the involution is defined as

$$
F^{*}(k, l, \tau)=\overline{F\left((k, l, \tau)^{-1}\right)}=\bar{F}\left(-k,-l, \bar{\tau} e^{2 \pi i \theta k \cdot l}\right) .
$$

Thus both $*_{\theta}$ and ${ }^{*}$ obviously depend on $\theta$.

The two convolutions $\natural_{\theta}$ and $*_{\theta}$ are closely related. Following [11, [15] we define an embedding $j$ of $\ell^{p}\left(\mathbb{Z}^{2 d}\right)$ into $L^{p}\left(\mathbb{H}_{\theta}\right)$ as follows: if $\mathbf{a} \in \ell^{p}$, then

$$
j(\mathbf{a})(k, l, \tau)=\bar{\tau} a(k, l) .
$$

To describe the range of $j$, we expand a function $F$ on $\mathbb{H}_{\theta}$ into a Fourier series with respect to the third coordinate $\tau$. Note that if $F \in L^{p}\left(\mathbb{H}_{\theta}\right)$, then for fixed $k, l \in \mathbb{Z}^{d}$ the function $\tau \rightarrow F(k, l, \tau)$ is in $L^{p}(\mathbb{T}) \subseteq L^{1}(\mathbb{T})$; therefore the Fourier coefficients $F_{n}(k, l)=\int_{\mathbb{T}} F(k, l, \tau) \bar{\tau}^{n} d \tau$ are well defined and the Fourier series

$$
F(k, l, \tau)=\sum_{-\infty}^{\infty} F_{n}(k, l) \tau^{n}
$$

converges in $L^{p}(\mathbb{T})$ for $1<p<\infty$.

The following lemma collects the properties of this embedding and clarifies the relation between $\ell^{p}\left(\mathbb{Z}^{2 d}\right)$ and $L^{p}\left(\mathbb{H}_{\theta}\right)$.

Lemma 2.3. (a) $j$ is a norm preserving ${ }^{*}$-homomorphism from $\left(\ell^{1}\left(\mathbb{Z}^{2 d}\right)\right.$, $\left.\natural_{\theta}\right)$ into $\left(L^{1}\left(\mathbb{H}_{\theta}\right), *_{\theta}\right)$ and an isometry from $\ell^{p}\left(\mathbb{Z}^{2 d}\right)$ into $L^{p}\left(\mathbb{H}_{\theta}\right)$ for $1<p \leq \infty$.

(b) The range of $j$ in $L^{p}(\mathbb{H})$ is $j\left(\ell^{p}\right)=\left\{F \in L^{p}: F_{n} \equiv 0\right.$ for $\left.n \neq-1\right\}$.

Next we describe the convolution on $L^{p}\left(\mathbb{H}_{\theta}\right)$ in terms of the expansion (2.5). 
Lemma 2.4. If $F \in L^{1}\left(\mathbb{H}_{\theta}\right)$ and $G \in L^{p}\left(\mathbb{H}_{\theta}\right)$, then

$$
\left(F *_{\theta} G\right)(m, n, \sigma)=\sum_{l=-\infty}^{\infty}\left(F_{l} \natural_{-\theta l} G_{l}\right)(m, n) \sigma^{l} .
$$

Proof. Since trigonometric polynomials are dense in $L^{p}(\mathbb{T}), 1 \leq p<\infty$, it is sufficient to prove (2.6) for functions $F$ and $G$ for which the expansion (2.5) is a finite series. The extension to all $F \in L^{1}\left(\mathbb{H}_{\theta}\right)$ and $G \in L^{p}\left(\mathbb{H}_{\theta}\right)$ follows from a routine density argument. We substitute the expansions for $F$ and $G$ into the definition of the group convolution and then use the orthogonality of $\tau^{n}$ on $L^{2}(\mathbb{T})$ to simplify:

$$
\begin{aligned}
(F & \left.*_{\theta} G\right)(m, n, \sigma) \\
& =\sum_{k, l \in \mathbb{Z}^{d}} \int_{\mathbb{T}} \sum_{r, s \in \mathbb{Z}} F_{r}(k, l) \tau^{r} G_{s}(m-k, n-l)\left(\sigma \bar{\tau} e^{2 \pi i \theta l \cdot(k-m)}\right)^{s} d \tau \\
& =\sum_{r \in \mathbb{Z}} \sum_{k, l \in \mathbb{Z}^{d}} \sigma^{r} F_{r}(k, l) G_{r}(m-k, n-l) e^{-2 \pi i \theta r(m-k) \cdot l} \\
& =\sum_{r \in \mathbb{Z}}\left(F_{r} \natural-\theta r G_{r}\right)(m, n) \sigma^{r},
\end{aligned}
$$

as announced.

Lemma 2.4 gives an idea why the irrational case $\theta \notin \mathbb{Q}$ is much more complicated than the rational case. For $\theta \in \mathbb{Q}$ only finitely many different twisted convolutions $\natural_{-\theta l}$ occur in the decomposition of $*_{\theta}$, whereas the case $\theta \notin \mathbb{Q}$ involves countably many (non-equivalent) twisted convolutions.

Corollary 2.5. The subspace $j\left(\ell^{p}\left(\mathbb{Z}^{2 d}\right)\right) \subseteq L^{p}\left(\mathbb{H}_{\theta}\right)$ is invariant under the action of $L^{1}\left(\mathbb{H}_{\theta}\right)$.

\subsection{Symmetric Group Algebras.}

Definition 2.6. An involutive Banach algebra $\mathcal{A}$ is called symmetric, if the spectrum of positive elements is positive, i.e., if $f=h^{*} h \in \mathcal{A}^{+}$, then $\sigma(f) \subseteq[0, \infty)$.

Moreover, $\mathcal{A}$ without identity element is symmetric, if and only if the algebra $\mathcal{A}_{1}$ obtained by adjoining an identity is symmetric [25] (4.7.9)].

An important tool to investigate the symmetry of an involutive Banach algebra is the following result of Hulanicki [16], which establishes a connection between the symmetry and the representation theory of $\mathcal{A}$.

Proposition 2.7 ([16]). Let $\mathcal{S}$ be a (not necessarily closed) *-subalgebra of an involutive Banach algebra $\mathcal{A}$. Suppose that there exists a faithful *-representation $(\pi, \mathcal{H})$ of $\mathcal{A}$ by bounded operators on a Hilbert space $\mathcal{H}$ such that for all $f=f^{*} \in \mathcal{S}$

$$
\|\pi(f)\|_{o p}=\lim _{n \rightarrow \infty}\left\|f^{n}\right\|_{\mathcal{A}}^{1 / n}=\nu_{\mathcal{A}}(f) .
$$

If $\mathcal{A}$ has an identity $e$, we assume that $\pi(e)=\operatorname{id}_{\mathcal{H}}$. Then for each $f=f^{*} \in \mathcal{S}$ we have

$$
\sigma_{\mathcal{A}}(f)=\sigma(\pi(f)) .
$$

In harmonic analysis it is of special interest to understand for which locally compact groups $G$ the group algebra $L^{1}(G)$ is symmetric. To get an impression of the depth and difficulty of this question the reader should consult [19], [21], 
[23], [20]. The symmetry of the group algebra has important consequences for the spectrum of convolution operators. If $f \in L^{1}(G)$, then the convolution operator $C_{f}$, defined as $C_{f} g=f * g$, is bounded on $L^{p}(G)$ for $1 \leq p \leq \infty$. Denote by $\sigma_{L^{p}}(f)$ the spectrum of $C_{f}$ acting on $L^{p}(G)$ and by $\nu_{L^{p}}(f)$ its spectral radius. If $f=f^{*}$, then $\nu_{L^{2}}(f)=\left\|C_{f}\right\|_{o p}$, the operator norm on $L^{2}(G)$, and $\nu_{L^{1}}(f)=$ $\lim _{n \rightarrow \infty}\|f * \cdots * f\|_{1}^{1 / n}$. In general the spectrum of $C_{f}$ will depend on the domain $L^{p}$. However, we have the following statement which is part of the folklore 1], 16, [19], [21], [23].

Theorem 2.8. Assume that $G$ is amenable. Then the following are equivalent.

(i) $L^{1}(G)$ is symmetric.

(ii) $\sigma_{L^{1}}(f)=\sigma_{L^{2}}(f)$ for all $f=f^{*} \in L^{1}(G)$.

Proof. The statement is well known; for completeness we give a short proof.

(ii) $\Longrightarrow$ (i): Assume that $\sigma_{L^{1}}(f)=\sigma_{L^{2}}(f)$ for $f=f^{*} \in L^{1}(G)$. Then $C_{f^{*} * f}=$ $C_{f}^{*} C_{f}$ is a positive operator on $L^{2}(G)$ and consequently $\sigma_{L^{2}}\left(f^{*} * f\right) \subseteq[0, \infty)$. Thus $\sigma_{L^{1}}\left(f^{*} * f\right) \subseteq[0, \infty)$ and so $L^{1}(G)$ is symmetric.

(i) $\Longrightarrow$ (ii) Suppose that $G$ is amenable, $L^{1}(G)$ is symmetric and $f=f^{*} \in L^{1}(G)$. Let $\lambda \in \sigma_{L^{1}}(f)$ be such that $|\lambda|=\nu_{L^{1}}(f)$. Then by [22, p. 311, V] there exists an irreducible $*$-representation $(\pi, \mathcal{H})$ of $L^{1}(G)$ on a Hilbert space $\mathcal{H}$ and a vector $\xi \in \mathcal{H},\|\xi\|=1$, such that $\lambda=\langle\pi(f) \xi, \xi\rangle$. Consequently

$$
\nu_{L^{1}}(f)=|\lambda| \leq\|\pi(f) \xi\|\|\xi\| \leq\|\pi(f)\|_{o p} .
$$

On the other hand, the amenability of $G[23$ implies that

$$
\|\pi(f)\|_{o p} \leq\left\|C_{f}\right\|_{o p}=\nu_{L^{2}}(f)
$$

and thus $\nu_{L^{1}}(f) \leq \nu_{L^{2}}(f)$. Since the converse inequality $\nu_{L^{2}}(f) \leq \nu_{L^{1}}(f)$ is always true, we have obtained the equality $\nu_{L^{2}}(f)=\nu_{L^{1}}(f)$. Now we apply Hulanicki's Lemma 2.7 to the regular representation $\pi(f)=C_{f}$ of $L^{1}(G)$ acting on $L^{2}(G)$ and obtain $\sigma_{L^{2}}(f)=\sigma_{L^{1}}(f)$ for all $f=f^{*} \in L^{1}(G)$.

Since all locally compact abelian groups are symmetric, one may expect that groups that are "similar" to abelian groups also have symmetric group algebras. This is indeed true for a large class of groups. For our purposes we need the following result of Ludwig [21].

Theorem 2.9. If $G$ is nilpotent, then $L^{1}(G)$ is symmetric. Consequently, $\sigma_{L^{1}}(f)=$ $\sigma_{L^{2}}(f)$ for $f=f^{*} \in L^{1}(G)$.

2.3. Wiener's Lemma for Twisted Convolution. By combining Theorem 2.9 with Proposition 2.7, it is now easy to prove our first version of Wiener's Lemma for twisted convolution. To distinguish twisted convolution from ordinary convolution, we write $L_{\mathbf{a}}$ for the twisted convolution operator $L_{\mathbf{a}} \mathbf{c}=\mathbf{a} \natural_{\theta} \mathbf{c}$, where $\mathbf{a} \in \ell^{1}\left(\mathbb{Z}^{2 d}\right)$ and $\mathbf{c} \in \ell^{p}\left(\mathbb{Z}^{2 d}\right)$. The spectrum of $L_{\mathbf{a}}$ acting on $\ell^{p}\left(\mathbb{Z}^{2 d}\right)$ is denoted by $\sigma_{\ell^{p}}(\mathbf{a})$; the spectral radius by $\nu_{\ell^{p}}(\mathbf{a})$. The identity element in $\ell^{1}\left(\mathbb{Z}^{2 d}\right)$ is denoted by $\delta$, where $\delta(0)=1$ and $\delta(k)=0$ for $k \neq 0$. We write $f^{n}$ and $\mathbf{a}^{n}$ for the $n$-th convolution power in $L^{1}(G)$ or $\ell^{1}\left(\mathbb{Z}^{2 d}\right)$.

Theorem 2.10. Assume that $\mathbf{a} \in \ell^{1}\left(\mathbb{Z}^{2 d}\right)$. Then

$$
\sigma_{\ell^{1}}(\mathbf{a})=\sigma_{\ell^{2}}(\mathbf{a}) \text {. }
$$


In particular, if $L_{\mathbf{a}}$ is invertible on $\ell^{2}\left(\mathbb{Z}^{2 d}\right)$, then $\mathbf{a}$ is invertible in $\ell^{1}\left(\mathbb{Z}^{2 d}\right)$ and there exists a unique $\mathbf{b} \in \ell^{1}\left(\mathbb{Z}^{2 d}\right)$ such that $L_{\mathbf{a}}^{-1}=L_{\mathbf{b}}$, or $\mathbf{a} \natural_{\theta} \mathbf{b}=\mathbf{b} \natural_{\theta} \mathbf{a}=\delta$. Consequently $L_{\mathbf{a}}$ is invertible simultaneously on all $\ell^{p}\left(\mathbb{Z}^{2 d}\right)$.

Proof. (a) We apply Hulanicki's Lemma to the algebra $\mathcal{A}=\mathcal{S}=\left(\ell^{1}\left(\mathbb{Z}^{2 d}\right), \natural_{\theta}, *\right)$ and the representation $\pi(\mathbf{a})=L_{\mathbf{a}}$ acting on $\ell^{2}\left(\mathbb{Z}^{2 d}\right)$. If $L_{\mathbf{a}}=0$, then $\mathbf{a}=\mathbf{a} \natural_{\theta} \delta=$ $L_{\mathbf{a}} \delta=0$; consequently $\pi$ is faithful.

In order to show that $\nu_{\ell^{1}}(\mathbf{a})=\nu_{\ell^{2}}(\mathbf{a})$, we use the identification of $\ell^{2}\left(\mathbb{Z}^{2 d}\right)$ with the closed subspace $j\left(\ell^{2}\right):=\mathcal{H}$ of $L^{2}\left(\mathbb{H}_{\theta}\right)$ and lift the arguments to $L^{1}\left(\mathbb{H}_{\theta}\right)$.

By Lemma 2.3 we have

$$
\nu_{\ell^{1}}(\mathbf{a})=\lim _{n \rightarrow \infty}\left\|\mathbf{a}^{n}\right\|_{1}^{1 / n}=\lim _{n \rightarrow \infty}\left\|j(\mathbf{a})^{n}\right\|_{1}^{1 / n}=\nu_{L^{1}}(j(\mathbf{a})) .
$$

On the invariant subspace $j\left(\ell^{2}\right) \subseteq L^{2}\left(\mathbb{H}_{\theta}\right)$ we have

$$
C_{j(\mathbf{a})} j(\mathbf{c})=j(\mathbf{a}) *_{\theta} j(\mathbf{c})=j\left(\mathbf{a} \natural_{\theta} \mathbf{c}\right)=j\left(L_{\mathbf{a}} \mathbf{c}\right),
$$

whereas on the orthogonal complement $j\left(\ell^{2}\right)^{\perp}$ we obtain that $\left.C_{j(\mathbf{a})}\right|_{j\left(\ell^{2}\right)^{\perp}}=0$ by Lemma 2.4. It follows that

$$
\left\|C_{j(\mathbf{a})}\right\|_{o p}=\left\|L_{\mathbf{a}}\right\|_{o p}=\nu_{\ell^{2}}(\mathbf{a})
$$

for any self-adjoint element $\mathbf{a}=\mathbf{a}^{*} \in \ell^{1}\left(\mathbb{Z}^{2 d}\right)$.

But since $L^{1}\left(\mathbb{H}_{\theta}\right)$ is symmetric by Theorem 2.9 we have that

$$
\nu_{L^{1}}(j(\mathbf{a}))=\nu_{L^{2}}(j(\mathbf{a}))=\left\|C_{j(\mathbf{a})}\right\|_{o p},
$$

and by means of (2.12) and (2.10) we conclude that $\nu_{\ell^{1}}(\mathbf{a})=\nu_{\ell^{2}}(\mathbf{a})$.

Thus the hypotheses of Proposition 2.7 are satisfied and we conclude that

$$
\sigma_{\ell^{1}}(\mathbf{a})=\sigma_{\ell^{2}}(\mathbf{a}) \quad \text { for } \mathbf{a}=\mathbf{a}^{*} \in \ell^{1}\left(\mathbb{Z}^{2 d}\right) .
$$

(b) If $\mathbf{a}, \mathbf{b} \in \ell^{1}\left(\mathbb{Z}^{2 d}\right)$ and $\mathbf{a} \natural_{\theta} \mathbf{b}=\mathbf{b} \natural_{\theta} \mathbf{a}=\delta$, then $L_{\mathbf{a}}^{-1}=L_{\mathbf{b}}$ is bounded on all $\ell^{p}\left(\mathbb{Z}^{2 d}\right), 1 \leq p \leq \infty$. Applying this observation to elements of the form $\mathbf{a}-\lambda \delta$ shows that

$$
\sigma_{\ell^{p}}(\mathbf{a}) \subseteq \sigma_{\ell^{1}}(\mathbf{a})
$$

for all $1 \leq p \leq \infty$.

(c) Next we show that

$$
\sigma_{\ell^{1}}(\mathbf{a})=\sigma_{\ell^{2}}(\mathbf{a}) \quad \text { for all } \mathbf{a} \in \ell^{1}\left(\mathbb{Z}^{2 d}\right) .
$$

If $\mathbf{a} \in \ell^{1}\left(\mathbb{Z}^{2 d}\right)$ and $L_{\mathbf{a}}$ is invertible on $\ell^{2}\left(\mathbb{Z}^{2 d}\right)$, then $L_{\mathbf{a}^{*}} \hbar_{\theta} \mathbf{a}$ is also invertible on $\ell^{2}\left(\mathbb{Z}^{2 d}\right)$. Since $\sigma_{\ell^{2}}\left(\mathbf{a}^{*} \bigsqcup_{\theta} \mathbf{a}\right)=\sigma_{\ell^{1}}\left(\mathbf{a}^{*} \natural_{\theta} \mathbf{a}\right)$ does not contain $0, \mathbf{a}^{*} \natural_{\theta} \mathbf{a}$ is invertible in $\ell^{1}\left(\mathbb{Z}^{2 d}\right)$. Likewise $\mathbf{a} \natural_{\theta} \mathbf{a}^{*}$ has an inverse in $\ell^{1}\left(\mathbb{Z}^{2 d}\right)$. This implies that $\mathbf{a}$ is invertible in $\ell^{1}\left(\mathbb{Z}^{2 d}\right)$, because it has the left inverse $\left(\mathbf{a}^{*} \natural_{\theta} \mathbf{a}\right)^{-1} \natural_{\theta} \mathbf{a}^{*} \in \ell^{1}\left(\mathbb{Z}^{2 d}\right)$ and the right inverse $\mathbf{a}^{*} \natural_{\theta}\left(\mathbf{a} \natural_{\theta} \mathbf{a}^{*}\right)^{-1} \in \ell^{1}\left(\mathbb{Z}^{2 d}\right)$. The argument applied to $\mathbf{a}-\lambda \delta$ shows that $\sigma_{\ell^{1}}(\mathbf{a}) \subseteq \sigma_{\ell^{2}}(\mathbf{a})$. Combined with (b) we obtain the identity of spectra (2.14).

(d) If $\mathbf{a} \in \ell^{1}\left(\mathbb{Z}^{2 d}\right)$ and $L_{\mathbf{a}}$ is invertible on $\ell^{2}\left(\mathbb{Z}^{2 d}\right)$, then $0 \notin \sigma_{\ell^{2}}(\mathbf{a})$ and (2.13) and (2.14) show that $0 \notin \sigma_{\ell^{p}}(\mathbf{a})$ for $1 \leq p \leq \infty$. Thus $L_{\mathbf{a}}$ is invertible on all $\ell^{p}\left(\mathbb{Z}^{2 d}\right)$.

Corollary 2.11. $\left(\ell^{1}\left(\mathbb{Z}^{2 d}\right), \natural_{\theta}{ }^{*}\right)$ is a symmetric Banach algebra. 
2.4. Weighted Convolution Algebras. In this section we extend Theorem 2.10 to weighted algebras.

By a weight $v$ on $\mathbb{R}^{2 d}$ we understand a positive, continuous, symmetric function that satisfies $v(0)=1$ and the submultiplicativity

$$
v\left(r_{1}+r_{2}\right) \leq v\left(r_{1}\right) v\left(r_{2}\right) \quad \text { for } r_{1}, r_{2} \in \mathbb{R}^{2 d} .
$$

The submultiplicativity (2.15) implies that $v$ grows at most exponentially, because $v(n r) \leq v(r)^{n}$ for all $r \in \mathbb{R}^{2 d}$ and $n \in \mathbb{N}$ and thus for some $\alpha>0$

$$
v(r) \leq\left(\max _{|e| \leq 1} v(e)\right)^{|r|}=e^{\alpha|r|}
$$

Let $\ell_{v}^{1}\left(\mathbb{Z}^{2 d}\right)$ be the space of all sequences on $\mathbb{Z}^{2 d}$ for which the norm

$$
\|\mathbf{a}\|_{1, v}=\sum_{r \in \mathbb{Z}^{2 d}}\left|a_{r}\right| v(r)
$$

is finite. Since $v$ is submultiplicative, we obtain

$$
\left\|\mathbf{a} \natural_{\theta} \mathbf{b}\right\|_{1, v} \leq\|\mathbf{a}\|_{1, v}\|\mathbf{b}\|_{1, v},
$$

and since $v$ is symmetric, we have $\left\|\mathbf{a}^{*}\right\|_{1, v}=\|\mathbf{a}\|_{1, v}$. Thus $\left(\ell_{v}^{1}\left(\mathbb{Z}^{2 d}\right), \natural_{\theta},{ }^{*}\right)$ is an involutive Banach algebra.

In the following we consider a special class of weight functions. Let $\rho$ be a norm on $\mathbb{R}^{2 d}$ (or on a lower-dimensional subspace $\neq\{0\}$ of $\mathbb{R}^{2 d}$ ), and let $\sigma$ be a non-negative, concave function on $\mathbb{R}^{+}$such that $\sigma(0)=0$ and

$$
\lim _{t \rightarrow \infty} \frac{\sigma(t)}{t}=0 .
$$

Definition 2.12. A weight $v$ is called subexponential if $v$ is of the form $v(r)=$ $e^{\sigma(\rho(r))}$.

Note that (2.18) is equivalent to the condition $\lim _{n \rightarrow \infty} v(n r)^{1 / n}=1$ for all $r \in \mathbb{R}^{2 d}$. This is the condition of Gelfand-Raikov-Shilov [12].

Example. Choose $c_{j}>0$ and a finite subset $F \subseteq\{1,2, \ldots, 2 d\}$. Then $\rho(r)=$ $\left(\sum_{j \in F}\left(\frac{r_{j}}{c_{j}}\right)^{p}\right)^{1 / p}$ is a norm on a subspace of $\mathbb{R}^{2 d}$. Choose $\sigma(t)=a \ln (1+t)$ or $\sigma(t)=a t^{b}, a>0,0 \leq b<1$. Thus the standard weight functions $v(r)=(1+|r|)^{a}$ and $v(r)=e^{a|r|^{b}}$ as well as "half-dimensional weights" $v(x, \omega)=(1+|x|)^{a}$ or $v(x, \omega)=(1+|\omega|)^{a}$ are covered by this definition.

Concerning the weighted convolution algebras $\ell_{v}^{1}\left(\mathbb{Z}^{2 d}\right)$ we have the following observation, which is inspired by a result of Pytlik [24].

Proposition 2.13. If $v$ is subexponential, then

$$
\nu_{\ell_{v}^{1}}(\mathbf{a})=\nu_{\ell^{1}}(\mathbf{a})
$$

for all $\mathbf{a} \in \ell_{v}^{1}\left(\mathbb{Z}^{2 d}\right)$.

Proof. We first define a sequence of auxiliary weights. Let

$$
\sigma_{n}(t)= \begin{cases}\sigma(t) & \text { if } t \geq n, \\ \frac{t}{n} \sigma(n) & \text { if } t \leq n\end{cases}
$$


Then $\sigma_{n}$ still satisfies (2.18) and is again positive and concave. It follows that $v_{n}(r)=e^{\sigma_{n}(\rho(r))}$ is a subexponential weight. Furthermore, there exist constants $a_{n}>0$ such that $\sigma_{n} \leq \sigma \leq \sigma_{n}+a_{n}$ and thus

$$
v_{n}(r) \leq v(r) \leq e^{a_{n}} v_{n}(r) .
$$

Since these weights are equivalent, we have $\ell_{v}^{1}\left(\mathbb{Z}^{2 d}\right)=\ell_{v_{n}}^{1}\left(\mathbb{Z}^{2 d}\right)$ and

$$
\nu_{\ell_{v_{n}}^{1}}(\mathbf{a})=\nu_{\ell_{v}^{1}}(\mathbf{a}) \quad \text { for } \mathbf{a} \in \ell_{v}^{1}\left(\mathbb{Z}^{2 d}\right) .
$$

Now we have

$$
\begin{aligned}
\nu_{\ell_{v}^{1}}(\mathbf{a})^{k} & =\left(\lim _{n \rightarrow \infty}\left\|\mathbf{a}^{k n}\right\|_{1, v}^{\frac{1}{k n}}\right)^{k} \\
& =\nu_{\ell_{v}^{1}}\left(\mathbf{a}^{k}\right) \\
& =\nu_{\ell_{v_{n}}^{1}}\left(\mathbf{a}^{k}\right) \quad \text { by }(\underline{2.22}) \\
& \leq\left\|\mathbf{a}^{k}\right\|_{1, v_{n}} \quad \text { for all } k \in \mathbb{N} .
\end{aligned}
$$

Since $v_{n} \rightarrow 1$ uniformly on compact sets and $v_{n} \leq v$, we obtain that

$$
\lim _{n \rightarrow \infty}\left\|\mathbf{a}^{k}\right\|_{1, v_{n}}=\lim _{n \rightarrow \infty}\left\|\mathbf{a}^{k} v_{n}\right\|_{1}=\left\|\mathbf{a}^{k}\right\|_{1}
$$

for all $k \in \mathbb{N}$. Combined with (2.23) we conclude that $\nu_{\ell_{v}^{1}}(\mathbf{a}) \leq\left\|\mathbf{a}^{k}\right\|_{1}^{1 / k}$ for all $k$, hence $\nu_{\ell_{v}^{1}}(\mathbf{a}) \leq \nu_{\ell^{1}}(\mathbf{a})$. Since the reverse inequality is always true, we have proved (2.19).

Theorem 2.14. If $\mathbf{a} \in \ell_{v}^{1}\left(\mathbb{Z}^{2 d}\right)$ and if $L_{\mathbf{a}}$ is invertible on $\ell^{2}\left(\mathbb{Z}^{2 d}\right)$, then there exists $\mathbf{b} \in \ell_{v}^{1}\left(\mathbb{Z}^{2 d}\right)$ such that $\mathbf{a} \natural_{\theta} \mathbf{b}=\mathbf{b} \natural_{\theta} \mathbf{a}=\delta$ whence $L_{\mathbf{a}}^{-1}=L_{\mathbf{b}}$. Consequently $L_{\mathbf{a}}$ is invertible simultaneously on all weighted spaces $\ell_{v}^{p}\left(\mathbb{Z}^{2 d}\right), 1 \leq p \leq \infty$.

Proof. We apply Proposition 2.7 to the Banach algebra $\ell_{v}^{1}\left(\mathbb{Z}^{2 d}\right) \subseteq \ell^{1}\left(\mathbb{Z}^{2 d}\right)$. Since by Theorem 2.10 and Proposition 2.13 we have

$$
\nu_{\ell_{v}^{1}}(\mathbf{a})=\nu_{\ell^{1}}(\mathbf{a})=\nu_{\ell^{2}}(\mathbf{a}),
$$

Proposition 2.7implies that $\sigma_{\ell_{v}^{1}}(\mathbf{a})=\sigma_{\ell^{2}}(\mathbf{a})$ for $\mathbf{a}=\mathbf{a}^{*} \in \ell_{v}^{1}\left(\mathbb{Z}^{2 d}\right)$. If $L_{\mathbf{a}}$ is invertible on $\ell^{2}$, then $0 \notin \sigma_{\ell^{2}}(\mathbf{a})$; thus $\mathbf{a}=\mathbf{a}^{*}$ is invertible in $\ell_{v}^{1}\left(\mathbb{Z}^{2 d}\right)$. Thus there exists $\mathbf{b} \in \ell_{v}^{1}\left(\mathbb{Z}^{2 d}\right)$ such that $\mathbf{a} \natural_{\theta} \mathbf{b}=\mathbf{b} \natural_{\theta} \mathbf{a}=\delta$. The extension to non-Hermitian elements follows as in step (c) of the proof of Theorem 2.10.

Corollary 2.15. If $v$ is a subexponential weight, then $\left(\ell_{v}^{1}\left(\mathbb{Z}^{2 d}\right), \natural_{\theta},{ }^{*}\right)$ is a symmetric Banach algebra.

Remark. This corollary is no longer true for exponential weights $v(r)=e^{\delta|r|}$. It follows from [12, pp. 152, 153] that the commutative algebra $\left(\ell_{v}^{1}\left(\mathbb{Z}^{2 d}\right), *\right)$ under ordinary convolution is not symmetric.

\section{Wiener's Lemma in the Rotation ALGEBRA}

For the applications to Gabor theory we study the representation $\pi$ of the algebra $\left(\ell^{1}\left(\mathbb{Z}^{2 d}\right), \natural_{\theta},{ }^{*}\right)$ acting on $L^{2}\left(\mathbb{R}^{d}\right)$ by time-frequency shifts. Recall that $\pi(\mathbf{a})$ is defined by

$$
\pi(\mathbf{a})=\sum_{k, l \in \mathbb{Z}^{d}} a_{k l} T_{\alpha k} M_{\beta l}
$$


Since $T_{\alpha k} M_{\beta l}$ is unitary, we have $\|\pi(\mathbf{a})\|_{o p} \leq\|\mathbf{a}\|_{1}$. By (1.14) and (2.1) $\pi$ is a nondegenerate representation of the involutive Banach algebra $\left(\ell_{v}^{1}\left(\mathbb{Z}^{2 d}\right), \natural_{\theta},{ }^{*}\right)$, where we have set $\alpha \beta=\theta$. Then $\pi\left(\ell_{v}^{1}\left(\mathbb{Z}^{2 d}\right)\right):=\mathcal{A}_{v}(\alpha, \beta)$ is a dense Banach subalgebra of the $C^{*}$-algebra $C^{*}(\alpha, \beta)$ that is generated by the time-frequency shifts $T_{\alpha k} M_{\beta l}$. Our main theorem is Wiener's Lemma for $\mathcal{A}_{v}(\alpha, \beta)$.

Theorem 3.1. Assume that $\mathbf{a} \in \ell_{v}^{1}\left(\mathbb{Z}^{2 d}\right)$ and that $\pi(\mathbf{a}) \in \mathcal{A}_{v}(\alpha, \beta)$ is invertible on $L^{2}\left(\mathbb{R}^{d}\right)$. Then $\mathbf{a}$ is invertible in $\ell_{v}^{1}\left(\mathbb{Z}^{2 d}\right)$, hence $\pi(\mathbf{a})^{-1}=\pi(\mathbf{b})$ for some $\mathbf{b} \in \ell_{v}^{1}\left(\mathbb{Z}^{2 d}\right)$ or $\pi(\mathbf{a})^{-1} \in \mathcal{A}_{v}(\alpha, \beta)$.

We prepare the proof of this theorem by several lemmas.

First we show that $\pi(\mathbf{a})$ is a meaningful operator for $\mathbf{a} \in \ell^{2}\left(\mathbb{Z}^{2 d}\right)$. For this purpose we introduce the amalgam space $W=W\left(L^{\infty}, \ell^{1}\right)$ on $\mathbb{R}^{d}$ with norm

$$
\|f\|_{W}=\sum_{k \in \mathbb{Z}^{d}} \sup _{x \in[0, \alpha]^{d}}|f(x+\alpha k)|=\sum_{k \in \mathbb{Z}^{d}}\left\|f \cdot T_{\alpha k} \chi_{[0, \alpha]^{d}}\right\|_{\infty} .
$$

Lemma 3.2. If $\mathbf{a} \in \ell^{2}\left(\mathbb{Z}^{2 d}\right)$, then $\pi(\mathbf{a})$ is bounded from $W\left(L^{\infty}, \ell^{1}\right)$ into $L^{2}\left(\mathbb{R}^{d}\right)$ and

$$
\|\pi(\mathbf{a}) f\|_{2} \leq C\|\mathbf{a}\|_{2}\|f\|_{W} .
$$

Proof. It suffices to establish (3.2) for elements a with compact support. Inequality (3.2) for arbitrary $\mathbf{a} \in \ell^{2}\left(\mathbb{Z}^{2 d}\right)$ then follows by density and continuity.

Write $\chi=\chi_{[0, \alpha]^{d}}$ and $f_{l}=f \cdot T_{\alpha l} \chi$. Then $f=\sum_{l \in \mathbb{Z}^{d}} f_{l}$ and $\|f\|_{W}=\sum_{l \in \mathbb{Z}^{d}}\left\|f_{l}\right\|_{\infty}$. Furthermore, by interchanging the order of $T_{\alpha k}$ and $M_{\beta l}$ we may rewrite $\pi(\mathbf{a})$ as

$$
\pi(\mathbf{a})=\sum_{k, l \in \mathbb{Z}^{d}} a_{k l} e^{-2 \pi i \theta k \cdot l} M_{\beta l} T_{\alpha k}=\sum_{k \in \mathbb{Z}^{d}} \mu_{k} T_{\alpha k},
$$

where $\mu_{k}(x)=\sum_{l \in \mathbb{Z}^{d}} a_{k l} e^{-2 \pi i \theta k \cdot l} e^{2 \pi i \beta l \cdot x}$ is $\frac{1}{\beta}$-periodic. If $\mathbf{a} \in \ell^{2}\left(\mathbb{Z}^{2 d}\right)$, then $\mu_{k}$ is locally square-integrable and for any compact set $K \subseteq \mathbb{R}^{d}$ and any $u \in \mathbb{R}^{d}$ we have

$$
\int_{u+K}\left|\mu_{k}(x)\right|^{2} d x \leq C \sum_{l \in \mathbb{Z}^{d}}\left|a_{k l}\right|^{2}
$$

with a constant independent of $\mathbf{a}$ and $u \in \mathbb{R}^{d}$. Using the decomposition of $f$ and $\left|f_{l}\right| \leq T_{\alpha l} \chi_{[0, \alpha]^{d}}\left\|f_{l}\right\|_{\infty}$, we can estimate $\|\pi(\mathbf{a}) f\|_{2}$ as follows:

$$
\begin{aligned}
\|\pi(\mathbf{a}) f\|_{2} & =\left\|\pi(\mathbf{a})\left(\sum_{l \in \mathbb{Z}^{d}} f_{l}\right)\right\|_{2} \leq \sum_{l \in \mathbb{Z}^{d}}\left\|\pi(\mathbf{a}) f_{l}\right\|_{2} \\
& =\sum_{l \in \mathbb{Z}^{d}}\left\|\sum_{k \in \mathbb{Z}^{d}} \mu_{k} T_{\alpha k} f_{l}\right\|_{2} \\
& \leq \sum_{l \in \mathbb{Z}^{d}}\left\|\sum_{k \in \mathbb{Z}^{d}}\left|\mu_{k}\right| T_{\alpha(k+l)} \chi\right\|_{2}\left\|f_{l}\right\|_{\infty} .
\end{aligned}
$$

Since the translates $T_{\alpha(k+l)} \chi$ are disjoint, (3.4) implies that

$$
\left\|\sum_{k \in \mathbb{Z}^{d}}\left|\mu_{k}\right| T_{\alpha(k+l)} \chi\right\|_{2}^{2}=\sum_{k \in \mathbb{Z}^{d}} \int_{\alpha(k+l)+[0, \alpha]^{d}}\left|\mu_{k}(x)\right|^{2} d x \leq C \sum_{k, n \in \mathbb{Z}^{d}}\left|a_{k n}\right|^{2} .
$$

Since this estimate is independent of $l$, (3.5) yields that

$$
\|\pi(\mathbf{a}) f\|_{2} \leq C\|\mathbf{a}\|_{2} \sum_{l \in \mathbb{Z}^{d}}\left\|f_{l}\right\|_{\infty}=C\|\mathbf{a}\|_{2}\|f\|_{W},
$$


which is the desired estimate.

Lemma 3.3. If $\mathbf{a} \in \ell^{2}\left(\mathbb{Z}^{2 d}\right)$ and $\pi(\mathbf{a})=0$ on $W\left(L^{\infty}, \ell^{1}\right)$, then $\mathbf{a}=0$.

Proof. We know from Lemma 3.2 that $\pi(\mathbf{a})$ is bounded on $W\left(L^{\infty}, \ell^{1}\right)$. Let $f, h$ be bounded functions with support in $[0, \alpha]^{d}$ (hence $f, h \in W$ ) and let $l, m \in \mathbb{Z}^{d}$ be arbitrary. The disjointness of the supports of $T_{\alpha k} f$ and (3.3) imply that

$$
\begin{aligned}
0 & =\left\langle\pi(\mathbf{a}) T_{\alpha l} f, T_{\alpha m} h\right\rangle \\
& =\sum_{k \in \mathbb{Z}^{d}}\left\langle\mu_{k} T_{\alpha(k+l)} f, T_{\alpha m} h\right\rangle \\
& =\left\langle\mu_{m-l} \cdot T_{\alpha m} f, T_{\alpha m} h\right\rangle .
\end{aligned}
$$

So we conclude that $\mu_{m-l}(x)=0$ for almost all $x \in \alpha m+[0, \alpha]^{d}$ and $l, m \in \mathbb{Z}^{d}$. Varying $l, m$, it follows that $\mu_{k}(x)=0$ for almost all $x \in \mathbb{R}^{d}$ and every $k \in \mathbb{Z}^{d}$. Consequently the Fourier coefficients of $\mu_{k}$ vanish and thus $\mathbf{a}=0$.

Lemma 3.4. For all $\mathbf{a} \in \ell^{1}\left(\mathbb{Z}^{2 d}\right)$ we have the inequalities

$$
\|\mathbf{a}\|_{2} \leq\left\|L_{\mathbf{a}}\right\|_{o p} \leq\|\mathbf{a}\|_{1}
$$

and

$$
\|\pi(\mathbf{a})\|_{o p} \leq\left\|L_{\mathbf{a}}\right\|_{o p} .
$$

Proof. Estimate (3.6) follows from

$$
\|\mathbf{a}\|_{2}=\left\|\mathbf{a} \natural_{\theta} \delta\right\|_{2} \leq\left\|L_{\mathbf{a}}\right\|_{o p}=\sup _{\|\mathbf{c}\|_{2}=1}\left\|\mathbf{a} \natural_{\theta} \mathbf{c}\right\|_{2} \leq\|\mathbf{a}\|_{1} .
$$

Estimate (3.7) is an easy consequence of Theorem 2.10] If $\mathbf{a}=\mathbf{a}^{*} \in \ell^{1}\left(\mathbb{Z}^{2 d}\right)$, then by (2.9) we have

$$
\begin{aligned}
\|\pi(\mathbf{a})\|_{o p} & =\nu(\pi(\mathbf{a})) \leq \nu_{\ell^{1}}(\mathbf{a}) \\
& =\nu_{\ell^{2}}(\mathbf{a})=\left\|L_{\mathbf{a}}\right\|_{o p} .
\end{aligned}
$$

Since for arbitrary a we have $\left\|\pi\left(\mathbf{a}^{*} \hbar_{\theta} \mathbf{a}\right)\right\|_{o p}=\left\|\pi(\mathbf{a})^{*} \pi(\mathbf{a})\right\|_{o p}=\|\pi(\mathbf{a})\|_{o p}^{2}$ and $\left\|L_{\mathbf{a}^{*} \mathfrak{\natural}_{\theta} \mathbf{a}}\right\|_{o p}=\left\|L_{\mathbf{a}}\right\|_{o p}^{2}$, (3.7) is proved for all $\mathbf{a} \in \ell^{1}\left(\mathbb{Z}^{2 d}\right)$.

Proof of Theorem 3.1. We wish to apply Proposition 2.7 to the subalgebra $\ell_{v}^{1}\left(\mathbb{Z}^{2 d}\right)$ of $\ell^{1}\left(\mathbb{Z}^{2 d}\right)$ and the representation $\pi$ on $L^{2}\left(\mathbb{R}^{d}\right)$. By Lemma $3.3 \pi$ is a faithful representation. Since we have already shown that $\nu_{\ell_{v}^{1}}(\mathbf{a})=\nu_{\ell^{2}}(\mathbf{a})=\left\|L_{\mathbf{a}}\right\|_{o p}$ for $\mathbf{a}=\mathbf{a}^{*} \in \ell_{v}^{1}\left(\mathbb{Z}^{2 d}\right)$ (Theorem 2.10 and Proposition 2.13), it suffices to verify that $\left\|L_{\mathbf{a}}\right\|_{o p}=\|\pi(\mathbf{a})\|_{o p}$.

Let $C^{*}\left(\ell^{1}\right)$ be the $C^{*}$-algebra generated by the regular (twisted) representation $L_{\mathbf{a}}$ of $\ell^{1}\left(\mathbb{Z}^{2 d}\right)$, and as above let $C^{*}(\alpha, \beta)$ be the $C^{*}$-algebra generated by the timefrequency shifts $T_{\alpha k} M_{\beta l}$.

If $A \in C^{*}\left(\ell^{1}\right)$, then there exists a sequence $\mathbf{a}_{n} \in \ell^{1}$ such that $\left\|A-L_{\mathbf{a}_{n}}\right\|_{o p} \rightarrow 0$. By (3.6) $\left\|\mathbf{a}_{n}-\mathbf{a}_{m}\right\|_{2} \leq\left\|L_{\mathbf{a}_{n}}-L_{\mathbf{a}_{m}}\right\|_{o p}$ and thus there exists $\mathbf{a} \in \ell^{2}$ such that $\lim \mathbf{a}_{n}=$ a. Because of (3.7) $\left\{\pi\left(\mathbf{a}_{n}\right)\right\}$ is a Cauchy sequence and thus there is $T \in C^{*}(\alpha, \beta)$ with $\left\|\pi\left(\mathbf{a}_{n}\right)-T\right\|_{o p} \rightarrow 0$. For $f \in W\left(L^{\infty}, \ell^{1}\right)$ we have both $\left\|\pi\left(\mathbf{a}_{n}\right) f-T f\right\|_{2} \rightarrow 0$ and $\left\|\pi\left(\mathbf{a}_{n}\right) f-\pi(\mathbf{a}) f\right\|_{2} \rightarrow 0$ by Lemma [3.2 so $T=\pi(\mathbf{a})$ on $W\left(L^{\infty}, \ell^{1}\right)$. By a similar argument we find that $A=L_{\mathbf{a}}$ on the dense subspace $\ell^{1}\left(\mathbb{Z}^{2 d}\right) \subseteq \ell^{2}\left(\mathbb{Z}^{2 d}\right)$.

Now we define a $C^{*}$-homomorphism $h$ from $C^{*}\left(\ell^{1}\right)$ to $C^{*}(\alpha, \beta)$ by

$$
h(A)=T,
$$


where $A$ and $T$ are as above. Then $h$ is a $*$-homomorphism, and by (3.7) and the construction $h$ is continuous. If $T=0$, then $\pi(\mathbf{a})=0$ on $W\left(L^{\infty}, \ell^{1}\right)$; consequently $\mathbf{a}=0$ by Lemma [3.3. Since $L_{\mathbf{a}} \mathbf{c}=A \mathbf{c}$ for all $\mathbf{c} \in \ell^{1}\left(\mathbb{Z}^{2 d}\right)$, we have $A=0$ and thus $h$ is one-to-one. Therefore we may conclude that $\left\|L_{\mathbf{a}}\right\|_{o p}=\|\pi(\mathbf{a})\|_{o p}$, because an injective $*$-homomorphism between $C^{*}$-algebras is always an isometry (see $[5$. Thm. I.5.5]).

In combination with Theorem 2.10 and Proposition 2.13 we have proved that

$$
\nu_{\ell_{v}^{1}}(\mathbf{a})=\nu_{\ell^{2}}(\mathbf{a})=\|\pi(\mathbf{a})\|_{o p} \quad \text { for } \mathbf{a} \in \ell_{v}^{1}\left(\mathbb{Z}^{2 d}\right) .
$$

Thus Proposition 2.7 implies that

$$
\sigma_{\ell_{v}^{1}}(\mathbf{a})=\sigma(\pi(\mathbf{a})) \quad \text { for } \mathbf{a}=\mathbf{a}^{*} .
$$

Consequently, if $\pi(\mathbf{a})$ is invertible on $L^{2}\left(\mathbb{R}^{d}\right)$, then $\mathbf{a}$ is invertible in the algebra $\ell_{v}^{1}\left(\mathbb{Z}^{2 d}\right)$, and thus there exists $\mathbf{b} \in \ell_{v}^{1}\left(\mathbb{Z}^{2 d}\right)$ such that $\mathbf{a} \natural_{\theta} \mathbf{b}=\mathbf{b} \natural_{\theta} \mathbf{a}=\delta$. Thus $\pi(\mathbf{b})=\pi(\mathbf{a})^{-1}$ as was to be proved. The extension to non-Hermitian elements follows as before.

Remark. Identity (3.10) was conjectured by Janssen in [17, (1.41)].

\section{Applichtions to Gabor frames And Window Design}

To establish the link between the abstract theory derived so far and timefrequency analysis on modulation spaces we need the following lemma. Recall that the modulation space $M_{v}^{1}$ is defined by the norm $\|f\|_{M_{v}^{1}}=\int_{\mathbb{R}^{2 d}}\left|V_{\phi} f(z)\right| v(z) d z$, where $V_{\phi} f$ is the short-time Fourier transform of $f$ with respect to the Gaussian window $\phi$ as defined in (1.3).

Lemma 4.1 ([14]). If $\mathbf{a} \in \ell_{\tilde{v}}^{1}\left(\mathbb{Z}^{2 d}\right)$, then $\pi(\mathbf{a})$ is bounded on $M_{v}^{1}$ and $\|\pi(\mathbf{a}) f\|_{M_{v}^{1}} \leq$ $\|\mathbf{a}\|_{1, \tilde{v}}\|f\|_{M_{v}^{1}}$, where $\tilde{v}$ is the restriction of $v$ to the lattice $\alpha \mathbb{Z}^{d} \times \beta \mathbb{Z}^{d}$.

Proof. A simple calculation shows that $\left|V_{\phi}\left(T_{u} M_{\eta} f\right)(x, \omega)\right|=\left|V_{\phi} f(x-u, \omega-\eta)\right|$. Because $v$ is submultiplicative, we obtain

$$
\left\|T_{u} M_{\eta} f\right\|_{M_{v}^{1}}=\int_{\mathbb{R}^{2 d}}\left|V_{\phi} f(x-u, \omega-\eta)\right| v(x, \omega) d x d \omega \leq v(u, \eta)\|f\|_{M_{v}^{1}} .
$$

Consequently

$$
\begin{aligned}
\|\pi(\mathbf{a}) f\|_{M_{v}^{1}} & =\left\|\sum_{k, l \in \mathbb{Z}^{d}} a_{k l} T_{\alpha k} M_{\beta l} f\right\|_{M_{v}^{1}} \\
& \leq \sum_{k, l \in \mathbb{Z}^{d}}\left|a_{k l}\right|\left\|T_{\alpha k} M_{\beta l} f\right\|_{M_{v}^{1}} \\
& \leq \sum_{k, l \in \mathbb{Z}^{d}}\left|a_{k l}\right| v(\alpha k, \beta l)\|f\|_{M_{v}^{1}}=\|\mathbf{a}\|_{1, \tilde{v}}\|f\|_{M_{v}^{1}} .
\end{aligned}
$$

Theorem 4.2. Assume that $\left\{T_{\alpha k} M_{\beta l} g: k, l \in \mathbb{Z}^{d}\right\}$ is a frame for $L^{2}\left(\mathbb{R}^{d}\right)$ and that $g \in M_{v}^{1}$. Then the Gabor frame operator $S_{g, \alpha, \beta}$ is invertible on $M_{v}^{1}$ and $\gamma=S^{-1} g \in M_{v}^{1}$. 
Proof. The so-called Janssen representation of the Gabor frame operator ([17] Prop. 2.8] and [4]) allows us to write $S$ as

$$
S=S_{g, \alpha, \beta}=(\alpha \beta)^{-d} \sum_{k, l \in \mathbb{Z}^{d}}\left\langle g, T_{\frac{k}{\beta}} M_{\frac{l}{\alpha}} g\right\rangle T_{\frac{k}{\beta}} M_{\frac{l}{\alpha}} .
$$

If $g \in M_{v}^{1}$, then it can be shown that $\sum_{k, l \in \mathbb{Z}^{d}}\left|\left\langle g, T_{k / \beta} M_{l / \alpha} g\right\rangle\right| v(k / \beta, l / \alpha)<\infty$; see [8] Lemma 7.2] and [14, Cor. 12.1.12]. Thus (4.1) can be recast by saying that $S=\pi(\mathbf{a})$ for some $\mathbf{a} \in \ell_{\tilde{v}}^{1}\left(\mathbb{Z}^{2 d}\right)$ with $\tilde{v}$ being the restriction of $v$ to $\frac{1}{\beta} \mathbb{Z}^{d} \times \frac{1}{\alpha} \mathbb{Z}^{d}$. Equivalently $S=S_{g, \alpha, \beta} \in \mathcal{A}_{\tilde{v}}\left(\frac{1}{\beta}, \frac{1}{\alpha}\right)$. Since $\left\{T_{\alpha k} M_{\beta l} g: k, l \in \mathbb{Z}^{d}\right\}$ is a frame, $S=\pi(\mathbf{a})$ is invertible. By Theorem 3.1. $S^{-1}=\pi(\mathbf{b}) \in \mathcal{A}_{\tilde{v}}\left(\frac{1}{\beta}, \frac{1}{\alpha}\right)$ and so $S^{-1}$ is of the form

$$
S^{-1}=\sum_{k, l \in \mathbb{Z}^{d}} b_{k l} T_{k / \beta} M_{l / \alpha} \quad \text { for } \mathbf{b} \in \ell_{\tilde{v}}^{1}\left(\mathbb{Z}^{2 d}\right) .
$$

By Lemma 4.1 $S^{-1}$ is bounded on $M_{v}^{1}$ and thus $\gamma=S^{-1} g \in M_{v}^{1}$.

We refer to [9, Section 5] and [14, Ch. 12] for the consequences of Theorem 4.2 for the time-frequency analysis of tempered distributions.

The next corollary confirms a conjecture of Janssen [18] in the irrational case. We say that a window $g$ satisfies condition (A) if

$$
\sum_{k, l \in \mathbb{Z}^{d}}\left|\left\langle g, T_{\frac{k}{\beta}} M_{\frac{l}{\alpha}} g\right\rangle\right|<\infty .
$$

This condition was introduced in 30 to avoid the obvious convergence problems of $S$ in (4.1). Since $S_{g, \alpha, \beta}^{-1}=S_{\gamma, \alpha, \beta}$ as a consequence of the computation

$$
\begin{aligned}
S_{g, \alpha, \beta}^{-1} f & =S^{-1} S S^{-1} f=\sum_{k, l \in \mathbb{Z}^{d}}\left\langle f, S^{-1} T_{\alpha k} M_{\beta l} g\right\rangle S^{-1} T_{\alpha k} M_{\beta l} g \\
& =\sum_{k, l \in \mathbb{Z}^{d}}\left\langle f, T_{\alpha k} M_{\beta l} \gamma\right\rangle T_{\alpha k} M_{\beta l} \gamma=S_{\gamma, \alpha, \beta} f,
\end{aligned}
$$

the question arose whether Janssen's representation of $S^{-1}$ also converges absolutely, or equivalently, whether $\gamma=S^{-1} g$ again satisfies condition (A). This was answered affirmatively by Janssen [18, Thm. 1.4] in the rational case $\alpha \beta \in \mathbb{Q}$ and conjectured to be true for all values of $\alpha \beta$. With the machinery built up so far we can now easily prove this conjecture.

Corollary 4.3. If $g$ satisfies condition ( $A$ ) and if the Gabor frame operator $S_{g, \alpha, \beta}$ is invertible, then $\gamma$ also satisfies condition $(A)$.

Proof. By definition condition (A) implies that $S_{g, \alpha, \beta}=\pi(\mathbf{a})$ for some $\mathbf{a} \in \ell^{1}$. Then Theorem 3.1 implies that $S^{-1}=\pi(\mathbf{b})$ for some $\mathbf{b} \in \ell^{1}$. Since $S^{-1}=S_{\gamma, \alpha, \beta}$, (4.1) and Lemma 3.3 imply that $b_{k l}=\left\langle\gamma, T_{k / \beta} M_{l / \alpha} \gamma\right\rangle$. Therefore $\gamma$ satisfies condition (A).

Here is another easy consequence of Theorem 3.1 Let $v_{s}(z)=(1+|z|)^{s}$ be the polynomial weight. Janssen [17, Prop. 5.4] showed that if $\pi(\mathbf{a}) \in \mathcal{A}_{v_{2 s+1}}$ is invertible on $L^{2}$, then $\pi(\mathbf{a})^{-1} \in \mathcal{A}_{v_{s}}$. We may now improve this statement as follows.

Corollary 4.4. If $\pi(\mathbf{a}) \in \mathcal{A}_{v_{s}}(\alpha, \beta)$ is invertible on $L^{2}\left(\mathbb{R}^{d}\right)$, then $\pi(\mathbf{a})^{-1} \in \mathcal{A}_{v_{s}}(\alpha, \beta)$. 
4.1. Tight Gabor Frames. For the construction of tight Gabor frames it is important to understand the mapping properties of the square root of $S_{g, \alpha, \beta}$. The interest stems from the following fact [3], [14]. Assume that $\left\{T_{\alpha k} M_{\beta l} g: k, l \in \mathbb{Z}^{d}\right\}$ is a frame for $L^{2}\left(\mathbb{R}^{d}\right)$, and define a new window $\tilde{\gamma}$ by $\tilde{\gamma}=S_{g, \alpha, \beta}^{-1 / 2} g$. Then every $f \in L^{2}\left(\mathbb{R}^{d}\right)$ has the non-orthogonal and unconditionally convergent expansion

$$
f=\sum_{k, l \in \mathbb{Z}^{d}}\left\langle f, T_{\alpha k} M_{\beta l} \tilde{\gamma}\right\rangle T_{\alpha k} M_{\beta l} \tilde{\gamma} .
$$

This so-called tight Gabor frame expansion works like an orthonormal expansion, but the coefficients are unique if and only if $\alpha \beta=1$ ([14. Cor. 7.5.2] and [28]). For genuine time-frequency analysis it is necessary that the series (4.3) converges in more restrictive norms, or equivalently that the window $\tilde{\gamma}$ is in $M_{v}^{1}$. The existence of tight frame expansions (4.3) with "good" windows follows easily from the existence of square roots of positive elements in involutive Banach algebras.

Corollary 4.5. (a) If $\pi(\mathbf{a}) \in \mathcal{A}_{v}(\alpha, \beta)$ is positive and invertible on $L^{2}\left(\mathbb{R}^{d}\right)$, then $\pi(\mathbf{a})^{ \pm 1 / 2} \in \mathcal{A}_{v}(\alpha, \beta)$.

(b) If $\left\{T_{\alpha k} M_{\beta l} g: k, l \in \mathbb{Z}^{d}\right\}$ is a frame for $L^{2}\left(\mathbb{R}^{d}\right)$ and $g \in M_{v}^{1}$, then $\tilde{\gamma}=$ $S_{g, \alpha, \beta}^{-1 / 2} g \in M_{v}^{1}$.

Proof. (a) Since $\sigma_{\ell_{v}^{1}}(\mathbf{a})=\sigma_{\ell^{2}}(\mathbf{a}) \subseteq(0, \infty)$, a is positive and invertible in the symmetric algebra $\ell_{v}^{1}\left(\mathbb{Z}^{2 d}\right)$. By [22, p. 305, VII] there exists a positive element $\mathbf{b}=\mathbf{b}^{*} \in$ $\ell_{v}^{1}\left(\mathbb{Z}^{2 d}\right)$ such that $\mathbf{a}=\mathbf{b} \natural_{\theta} \mathbf{b}$. Consequently $\pi(\mathbf{b})^{2}=\pi(\mathbf{a})$ and $\pi(\mathbf{b}) \in \mathcal{A}_{v}(\alpha, \beta)$. By construction $0 \notin \sigma(\pi(\mathbf{b}))$ and thus $\pi(\mathbf{b})^{-1} \in \mathcal{A}_{v}(\alpha, \beta)$ as well.

(b) follows by applying (a) to the positive invertible operator $S_{g, \alpha, \beta}^{-1} \in \mathcal{A}_{\tilde{v}}\left(\frac{1}{\beta}, \frac{1}{\alpha}\right)$.

4.2. Generalizations. Instead of the "separable" lattice $\alpha \mathbb{Z}^{d} \times \beta \mathbb{Z}^{d}$ one may consider arbitrary "non-separable" lattices $\Lambda=A \mathbb{Z}^{2 d}$ for some $A \in G L(2 d, \mathbb{R})$. Write $z=\left(z_{1}, z_{2}\right) \in \mathbb{R}^{d} \times \mathbb{R}^{d}=\mathbb{R}^{2 d}$ and $U_{z}=T_{z_{1}} M_{z_{2}}$ for the time-frequency shifts, and define the cocycle $\chi(z, w)=e^{2 \pi i w_{1} \cdot z_{2}}$. Then the twisted convolution on $\ell^{1}(\Lambda)$ is defined to be

$$
\left(\mathbf{a} \natural_{\Lambda} \mathbf{b}\right)(\mu)=\sum_{\lambda \in \Lambda} a_{\lambda} b_{\mu-\lambda} \chi(\lambda, \mu-\lambda)
$$

for $\mathbf{a}, \mathbf{b} \in \ell^{1}(\Lambda)$. It can be shown that the operators

$$
\pi_{\Lambda}(\mathbf{a})=\sum_{\lambda \in \Lambda} a_{\lambda} U_{\lambda}
$$

define a faithful non-degenerate representation of $\ell^{1}(\Lambda)$ on $L^{2}\left(\mathbb{R}^{d}\right)$. Then the noncommutative versions of Wiener's Lemma, Theorems 2.10 2.14 and 3.1 extend to the algebra $\left(\ell^{1}(\Lambda), \natural_{\Lambda}\right)$ with a suitable involution. In particular, if $\pi_{\Lambda}(\mathbf{a})$ is invertible on $L^{2}\left(\mathbb{R}^{d}\right)$ and if $\mathbf{a} \in \ell_{v}^{1}(\Lambda)$, then $\pi_{\Lambda}(\mathbf{a})^{-1}=\pi_{\Lambda}(\mathbf{b})$ for some $\mathbf{b} \in \ell_{v}^{1}(\Lambda)$. Likewise, the other results can be generalized to general lattices in the time-frequency plane. The proofs are almost identical and the minor modifications are left to the reader.

In a further generalization we may replace $\mathbb{R}^{d}$ by an arbitrary second countable locally compact abelian group $G$ and $\Lambda \subseteq \mathbb{R}^{2 d}$ by a discrete cocompact subgroup $D \subseteq G \times \widehat{G}$. Time-frequency shifts on $L^{2}(G)$ are defined by $T_{x} M_{\chi} f(t)=$ $\chi(t-x) f(t-x)$ for $x, t \in G$ and $\chi \in \widehat{G}$. The abstract structures are the same, and all results of this paper can be formulated and proved for twisted convolution on 
$D$ and for the corresponding projective representation $\pi_{D}$ of $D$ by time-frequency shifts on $L^{2}(G)$. As no new ideas or techniques are required, we omit the discussion of details.

\section{ACKNOWLEDGEMENT}

Research on this paper was done while the first named author was Visiting Professor at the Department of Mathematics at the Universities of Heidelberg and Vienna. He would like to express his gratitude for the hospitality and generosity during his stay.

\section{REFERENCES}

[1] B. A. Barnes. When is the spectrum of a convolution operator on $L^{p}$ independent of $p$ ? Proc. Edinburgh Math. Soc. (2), 33(2):327-332, 1990. MR 91f:47046

[2] H. Bölcskei and A. J. E. M. Janssen. Gabor frames, unimodularity, and window decay. J. Fourier Anal. Appl., 6(3):255-276, 2000. MR 2001b:42044

[3] I. Daubechies. The wavelet transform, time-frequency localization and signal analysis. IEEE Trans. Inform. Theory, 36(5):961-1005, 1990. MR 91e:42038

[4] I. Daubechies, H. J. Landau, and Z. Landau. Gabor time-frequency lattices and the WexlerRaz identity. J. Fourier Anal. Appl., 1(4):437-478, 1995. MR 96i:42021

[5] K. R. Davidson. $C^{*}$-algebras by example. American Mathematical Society, Providence, RI, 1996. MR 97i:46095

[6] G. A. Elliott and D. E. Evans. The structure of the irrational rotation $C^{*}$-algebra. Ann. of Math. (2), 138(3):477-501, 1993. MR 94j:46066

[7] H. G. Feichtinger. On a new Segal algebra. Monatsh. Math., 92(4):269-289, 1981. MR 83a:43002

[8] H. G. Feichtinger and K. Gröchenig. Banach spaces related to integrable group representations and their atomic decompositions. II. Monatsh. Math., 108(2-3):129-148, 1989. MR 91g:43012

[9] H. G. Feichtinger and K. Gröchenig. Gabor frames and time-frequency analysis of distributions. J. Functional Anal., 146(2):464-495, 1997. MR 98k:42041

[10] H. G. Feichtinger and T. Strohmer, editors. Gabor analysis and algorithms: theory and applications. Birkhäuser Boston, Boston, MA, 1998. MR 98h:42001

[11] G. B. Folland. Harmonic Analysis in Phase Space. Princeton Univ. Press, Princeton, NJ, 1989. MR 92k:22017

[12] I. Gel'fand, D. Raikov, and G. Shilov. Commutative normed rings. Chelsea Publishing Co., New York, 1964. MR 34:4940

[13] K. Gröchenig. An uncertainty principle related to the Poisson summation formula. Studia Math., 121(1):87-104, 1996. MR 98a:42007

[14] K. Gröchenig. Foundations of time-frequency analysis. Birkhäuser Boston Inc., Boston, MA, 2001. MR 2002h:42001

[15] R. Howe. On the role of the Heisenberg group in harmonic analysis. Bull. Amer. Math. Soc. (N.S.), 3(2):821-843, 1980. MR 81h:22010

[16] A. Hulanicki. On the spectrum of convolution operators on groups with polynomial growth. Invent. Math., 17:135-142, 1972. MR 48:2304

[17] A. J. E. M. Janssen. Duality and biorthogonality for Weyl-Heisenberg frames. J. Fourier Anal. Appl., 1(4):403-436, 1995. MR 97e:42007

[18] A. J. E. M. Janssen. On rationally oversampled Weyl-Heisenberg frames. Signal Proc., 47:239$245,1995$.

[19] H. Leptin. The structure of $L^{1}(G)$ for locally compact groups. In Operator algebras and group representations, Vol. II (Neptun, 1980), pages 48-61. Pitman, Boston, Mass., 1984. MR 85d:22015

[20] V. Losert. On the structure of groups with polynomial growth. II. J. London Math. Soc. (2), 63(3):640-654, 2001. MR 2002f:22007

[21] J. Ludwig. A class of symmetric and a class of Wiener group algebras. J. Funct. Anal., 31(2):187-194, 1979. MR 81a:43007 
[22] M. A. Naŭmark. Normed algebras. Wolters-Noordhoff Publishing, Groningen, third edition, 1972. Translated from the second Russian edition by Leo F. Boron, Wolters-Noordhoff Series of Monographs and Textbooks on Pure and Applied Mathematics. MR 55:11042

[23] A. L. T. Paterson. Amenability. American Mathematical Society, Providence, RI, 1988. MR 90e: 43001

[24] T. Pytlik. On the spectral radius of elements in group algebras. Bull. Acad. Polon. Sci. Sér. Sci. Math. Astronom. Phys., 21:899-902, 1973. MR 48:6818

[25] C. E. Rickart. General theory of Banach algebras. D. van Nostrand Co., Inc., Princeton, N.J.-Toronto-London-New York, 1960. The University Series in Higher Mathematics. MR 22:5903

[26] M. A. Rieffel. Von Neumann algebras associated with pairs of lattices in Lie groups. Math. Ann., 257(4):403-418, 1981. MR 84f:22010

[27] M. A. Rieffel. Projective modules over higher-dimensional noncommutative tori. Canad. J. Math., 40(2):257-338, 1988. MR 89m:46110

[28] A. Ron and Z. Shen. Weyl-Heisenberg frames and Riesz bases in $L_{2}\left(\mathbb{R}^{d}\right)$. Duke Math. J., 89(2):237-282, 1997. MR 98i:42013

[29] T. Strohmer. Approximation of dual Gabor frames, window decay, and wireless communications. Appl. Comput. Harmon. Anal., 11(2):243-262, 2001. MR 2002j:42049

[30] R. Tolimieri and R. S. Orr. Poisson summation, the ambiguity function, and the theory of Weyl-Heisenberg frames. J. Fourier Anal. Appl., 1(3):233-247, 1995. MR 97c:94001

[31] D. F. Walnut. Continuity properties of the Gabor frame operator. J. Math. Anal. Appl., 165(2):479-504, 1992. MR 93f:42059

[32] M. Zibulski and Y. Y. Zeevi. Analysis of multiwindow Gabor-type schemes by frame methods. Appl. Comput. Harmon. Anal., 4(2):188-221, 1997. MR 98f:42035

Department of Mathematics, The University of Connecticut, Storrs, CT 06269-3009 E-mail address: GROCH@MATH.UCONN.EDU

Institut für Angewandte Mathematik, Fakultät für Mathematik, Im Neuenheimer Feld 288, D-69120 Heidelberg, Germany

E-mail address: LEINERT@MATH.UNI-HEIDELBERG.DE 